\title{
A review of the British Thoracic Society scientific meeting 1-3 December 2010
}

\author{
Colin Church, Hannah K Bayes, Andrew J Fisher, On behalf of the BTS Science and \\ Research Committee
}

BTS Science and Research Committee, The British Thoracic Society, 17 Doughty Street, London WC1N 2PL; UK

\section{Correspondence to Professor Andrew J Fisher, Institute of Cellular Medicine, Newcastle University Medical School, Framlington Place, Newcastle Upon Tyne NE2 4HH, UK; a.j.fisher@ncl.ac.uk}

CC and HKB contributed equally to this work.

Received 20 December 2010 Accepted 21 December 2010 Published Online First 17 January 2011

\section{ABSTRACT}

This review presents the inaugural report of the British Thoracic Society Winter Scientific Meeting held from $1^{\text {st }}-3^{\text {rd }}$ December 2010. Although a wide spectrum of respiratory research was presented at the meeting the content of the review focuses specifically on three key themes of inflammation and lung injury, airways disease and respiratory infection. Advances in both clinical and translational respiratory research presented within the major symposia and spoken sessions related to these themes are summarised.

The British Thoracic Society (BTS) Winter Scientific Meeting provides an important interface between advances in the basic science of lung disease, translational and clinical respiratory research and clinical respiratory medicine. The meeting provides the platform for the presentation of new knowledge and new developments to the greater respiratory community. The volume and standard of the work presented at the BTS Winter Meeting continues to increase year on year. In addition to a large number of peer-reviewed abstracts and invited symposium speakers, this year the BTS welcomed a number of outstanding keynote speakers including Professor Jeffrey Drazen, editor of the New England Journal of Medicine and Professor Marc Decramer, President of the European Respiratory Society.

Thorax is pleased to present this inaugural review of the meeting, in recognition of the role it plays in the respiratory calendar. It would be impossible, however, to include the entire meeting content in this review, and so three key areas have been identified and the review will focus on the symposia and spoken abstracts within those fields, namely inflammation and lung injury, airways disease and respiratory infection.

\section{'DANGER SIGNALS' AND LUNG INJURY}

This year the joint BTS/British Association of Lung Research (BALR) session provided a two-part symposium on the science and role of 'danger signals' within respiratory disease. 'Danger signals' within the respiratory system are initiated by environmental, infectious or endogenous factors. Innate immune responses to 'danger' are initiated by pattern recognition receptors (PRRs) which recognise molecules that are evolutionarily conserved and broadly shared by pathogens; collectively these are referred to as pathogen-associated molecular patterns or, in the case of non-infectious agents, damageassociated molecular patterns. An excellent overview of the roles of the PRRs, Toll-like receptors (TLRs) and the cytosolic NOD-like receptors in sensing pathogens and as initiators of respiratory inflammation was given by Professor L O'Neill (Dublin). ${ }^{1}$ Professor G Brown (Aberdeen) also stressed the important role that the C-type lectins play in triggering innate responses to fungal infection. ${ }^{3}$

Engagement of these PRRs initiates intracellular signalling pathways that ultimately orchestrate a range of cellular and cytokine/chemokine responses that direct the course of the adaptive immune response. Assembly of large intracellular caspase-1activating complexes, called inflammasomes, represents an important response to certain pathogen-associated molecular patterns. Activation of caspase-1 leads to processing and secretion of the proinflammatory cytokine interleukin $1 \beta$ (IL-1 $\beta$ ) which has been linked to the pathogenesis of both acute and chronic lung inflammation. Professor I Couillin (Orleans, France) discussed the important emerging respiratory 'danger' of nanoparticles. The unusual physical and chemical properties of nanoparticles allows them to access the cell cytosol, without the need for phagocytosis, where they can activate the inflammasone. Professor Couillin's group demonstrate that nanoparticles induce lung inflammation in vivo via both IL- $1 \alpha$ and IL-1 $\beta$, and partial activation of the NLRP3 inflammasone. ${ }^{4}$

Respiratory allergens that are capable of activating PRRs and TLR4 polymorphisms have been linked to allergic asthma. Professor B Lambrecht (Ghent, Belgium) highlighted the role of TLR4 in initiating and perpetuating airways inflammation in response to house dust mite protein, ${ }^{5}$ and the effect of the endogenous danger signal uric acid in amplifying this response. Separately, in a spoken session, Cottey and colleagues reported upregulation of epithelial gene expression for TLR2 and TLR4 in asthmatic airways, whereas TLR5 appeared to be downregulated in patients with more severe, steroid-treated asthma. ${ }^{6}$ Thus TLR antagonism may represent a therapeutic option in airways hypersensitivity.

The role of 'danger signals' in chronic lung fibrosis was highlighted by Professor P Noble (Durham, USA), who presented his extensive work on the role of the extracellular matrix glycosaminoglcan hyaluronan in inducing lung inflammation and fibrosis. ${ }^{7} 8$ Fragmented hyaluronan induces innate immune responses via TLR2 and TLR4. Hyaluronan is ubiquitiously expressed throughout the lung, yet the role this plays in protection versus inflammation and fibrogenesis appears to be location dependent.

The theme of lung injury was extended in several sessions dedicated to the pathogenesis and 
treatment of acute lung injury (ALI). ALI continues to carry significant mortality, but proven pharmacological treatments remain elusive and novel therapeutic targets continue to be explored. In this year's BTS lecture, Professor M Matthay (San Francisco) highlighted the therapeutic potential of stem cellbased therapy in ALI. Excellent spoken sessions also addressed mediators and novel targets in ALI. Shyamsundar et al reported an in vivo trial of keratinocyte growth factor (KGF) in a human lipopolysaccaride model of ALI. ${ }^{9}$ The authors suggest that, although the pulmonary inflammatory infiltrate and permeability was unchanged by KGF pretreatment, the resultant upregulation of factors implicated in epithelial repair supports a potential therapeutic role for KGF. A potential role for vitamin $\mathrm{D}$ was highlighted by Dancer et al, with patients with ALI having low levels of the vitamin, which correlated with mediators of systemic inflammatory response and survival. ${ }^{10}$ They demonstrated that T cell proinflammatory IL-17 production was upregulated at the onset of ALI and could be attenuated by exogenous 1,25 -hydroxyvitamin $\mathrm{D} .{ }^{11}$ The authors suggest that a therapeutic trial of vitamin D in ALI is warranted.

The important role of PRRs was also highlighted in ALI via the involvement of the receptor for advanced glycation endproducts (RAGEs). ${ }^{12}$ RAGE ligands are elevated in patients with systemic inflammatory response syndrome (SIRS) and induce proinflammatory responses. Thompson et al presented in vitro evidence that RAGE ligation increases bronchial endothelial IL-8 secretion and reduces antiproteases, effects that were attenuated by soluble RAGEs or RAGE-blocking antibodies. ${ }^{13}$ Zakeri et al were joint winners of the BTS Medical Student Abstract Prize for a case-control study of RAGE polymorphisms in 187 patients undergoing cardiac surgery requiring cardiopulmonary bypass. ${ }^{14}$ The authors demonstrated an association between single nucleotide polymorphisms in the RAGE and the RAGE ligand, S100A8, genes and the intensity of the systemic inflammatory response and lung injury. Therapeutic targeting of this PRR in ALI may thus represent a means of attenuating damaging inflammation, potentially directing preventative treatment based on patient genotype.

\section{INFLAMMATORY AIRWAYS DISEASE}

It recognition of the 25th anniversary of the British Lung Foundation (BLF), Professor S Buist (Washington, USA) gave a compelling talk on the epidemiology of chronic obstructive pulmonary disease (COPD) and showed how it will soon become the western world's third leading cause of death. Worryingly, mortality is still increasing in females in comparison with males, in which it has now reached a plateau. Her lecture went on to detail the risk factors across the world for this disease and introduced the concept of COPD being a result of cumulative cellular damage from environmental insults acting on disease-specific genes spanning from prenatal to elderly years. The epidemiology of airways disease was further mentioned in an interesting abstract by Palmer et al. ${ }^{15}$ This joint BTS medical student prize-winning talk showed how, after adjusting for covariants including smoking, substance misusers seem to have higher prevalence of respiratory disease including COPD. It is unclear why this should be and deserves further investigation.

Several sessions addressed the interaction between the environment and inflammatory responses in COPD. Moisey et al showed how both primary bronchial epithelial cells and macrophages exposed to cigarette smoke extract (CSE) and Haemophilus influenzae led to an altered and proneutrophilic inflammatory response. suggesting this as a mechanism for ongoing inflammation in COPD airways. ${ }^{16}$ In addition, Comer et al demonstrated how CSE can alter the innate immune response via altered expression of TLR4 on nasal epithelial cells. ${ }^{17}$ Meanwhile in a talk examining the vascular changes and lower levels of vascular endothelial growth factor (VEGF) associated with emphysema by Mackay et al, microvascular endothelial cells isolated from patients with emphysema were shown to undergo accelerated apoptosis and downregulate VEGF receptor 2 in response to CSE. ${ }^{18}$ These findings could suggest the dysregulation of both inflammatory and microvascular function in patients with emphysema.

In the symposium examining 'COPD: the patient with complex advanced disease' there was an interesting and detailed discussion by Professor A Agusti (Barcelona) on the importance of treating both the airways disease and systemic manifestations of this disease process. ${ }^{19-21} \mathrm{He}$ clearly showed that COPD is a heterogeneous disease with pulmonary and extrapulmonary manifestations and that a better understanding of this complexity is essential for the adequate risk stratification of these patients, as well as for the development of novel and specifically targeted treatments. This was followed by Dr C Ruse (Sheffield) explaining the difficulties encountered by physicians in treating elderly patients with COPD and recommending that Care of the Elderly physicians should be involved at an early stage in order to try and optimise the best placement for these patients. Professor M Polkey (London) discussed the role of volume reduction surgery, highlighting the importance of patient selection for predominantly upper lobe disease. He then discussed the exciting development of the use of endobronchial valves in heterogenous emphysema and the improvement in functionality and forced expiratory volume in $1 \mathrm{~s}\left(\mathrm{FEV}_{1}\right)$ seen in the VENT trial. ${ }^{22}$ Further studies are required to demonstrate the clinical usefulness and practicality of this technique.

Dr M Elliot (Leeds) led an interesting talk on the potential role of domiciliary non-invasive ventilation (NIV) in patients with COPD. This is a relatively new area of research and, as such, the evidence base is currently low. He suggests current recommendations would be to use domiciliary NIV in patients who have symptomatic hypercapnia on long-term oxygen therapy or possibly in those patients who despite maximal therapy are having recurrent admissions to hospital with non-infective type 2 respiratory failure. ${ }^{23}$ In cases other than these, patients should probably only commence long-term NIV in the context of a clinical trial.

A tremendous journey through the pathobiology of $\alpha$-1-antitrypsin (AAT-1) was given by Professor D Lomas (Cambridge) in the plenary scientific symposium. The dysfunctional polymerisation of these protein monomers into larger aggregates results in neutrophil recruitment and activation of proinflammatory responses. This process of polymerisation also seems to be driven by exposure to cigarette smoke. ${ }^{24} \mathrm{He}$ also explored the potential of in vitro differentiation of fibroblasts from patients with AAT-1 deficiency into hepatocytes, which could then be utilised to study the molecular characteristics of the underlying hepatic dysfunction. ${ }^{25}{ }^{26}$ Finally, he discussed the promise that genome-wide association studies have in identifying key genes which may provide a key to unlocking the mechanisms of COPD. ${ }^{27}$ In addition, such studies may answer why within families there is a predominant form of COPD phenotype clustering and why some smokers are more prone to COPD development than others.

Linking with the symposium 'COPD: the patient with complex advanced disease', an excellent spoken session further examined survival in this disease. In keeping with the multisystem nature of COPD, Rashid et al studied the association of red cell indices with survival. ${ }^{28}$ In their cohort, $10 \%$ of patients 
demonstrated iron deficiency, and this was associated with poorer survival, as was red cell distribution width. Such information may suggest a therapeutic intervention in COPD as well as aid prognostication. In the general population, obesity is widely associated with decreased life expectancy. ${ }^{29}{ }^{30}$ In contrast, Collins et al studied $>400$ outpatients with COPD and demonstrated that overweight/obese patients had lower rates of hospital admission and mortality at 1 year compared with normal or underweight patients. ${ }^{31}$ The same group demonstrated that deprivation is significantly associated with higher 1-year mortality in outpatients with COPD independent of disease severity, malnutrition and age. ${ }^{32}$

Novel data in the field of asthma were reflected in numerous symposia and abstracts. Shikotra et al looked at a relatively newcomer to the cytokine mileau of asthma, namely thymic stromal lymphopoetin. ${ }^{33}$ Using immunohistochemistry they found increased staining of thymic stromal lymphopoetin in the epithelial cells in biopsies from patients with mild and severe asthma compared with those without asthma. This may point to an important role in airways disease. ${ }^{34}$ In the session addressing the interface between airways inflammation and remodelling, the role of accelerated apoptosis leading to faster resolution of eosinophilic lung inflammation was explored. Farahi et al showed in vitro that pan-inhibition of cyclin-dependent kinases in eosinophils by a novel compound R-roscovitine led to their increased apoptosis and necrosis with subsequent phagocytosis by macrophages. However, reflecting the often found differences between in vitro and in vivo work, when this compound was applied to a murine model of asthma there was no reduction seen in eosinophil infiltrates in the blood or lung tissue. ${ }^{35}$

The importance of histamine release from lung mast cells in airways biology is fundamental. Martin et al investigated further the observation that direct interaction between epithelial and mast cells prevents constitutive and immunoglobulin E (IgE)mediated mast cell degranulation. ${ }^{36}$ By using a co-culture technique, in which cells were in close proximity but not adherent, they showed that an, as yet unidentified, soluble mediator secreted by the epithelial cells can inhibit histamine release from mast cells. This was not seen in epithelial cells derived from patients with asthma, suggesting a loss of this factor in the asthma phenotype. Its identification may lead to novel therapeutic avenues.

Of course understanding the basic mechanisms of disease is important, but this must be translated into efficacious patient treatments. The nature of fungal disease in the context of asthma was investigated by Agbetile et al. ${ }^{37}$ They looked at 126 patients with asthma and, using a more sensitive fungal culture technique, found that $>60 \%$ cultured a mould in their sputum (Aspergillus being the most common isolate). They also demonstrated that the $\mathrm{FEV}_{1}$ was significantly lower in those patients who were fungal positive on culture versus those who were negative, hinting that fungal colonistion may be a driving force for airways disease.

The management of difficult asthma was the subject of a well attended symposium and dealt with wide-ranging issues. Dr R Niven (Manchester) delivered a talk which outlined the definition of difficult asthma as persistent symptoms with frequent exacerbations despite prescribed treatment at BTS step 4 or 5 , albeit that this definition lacks worldwide consensus. He went on to point out the many risk factors for this, including those who have predominantly neutrophilic airways inflammation and mould sensitisation. Finally he mentioned the BTS Difficult Asthma network which is a UK-wide collaboration between centres specialising in difficult asthma and will allow further detailed analysis of the characteristics of patients attending these clinics. ${ }^{38}$ Dr P Gill (Sheffield) followed on from this, suggesting that liaison psychiatry should play a larger role in the management of this challenging illness and that we should ignore the psychological component at our peril. Dr L Heaney (Belfast) emphasised the essential role of patient adherence to their medication. ${ }^{39}$ He elegantly showed that as many as $35 \%$ of patients with severe asthma may not actually be taking their medication even though they might claim to during consultation with the doctor. Using a health cost analysis, it was estimated that if we checked and encouraged these patients to take their medication it may save up to a staggering $£ 43$ million/year in unnecessary prescribing of expensive medication, such as anti-IgE therapy. Professor I Pavord (Leicester) dealt with the issue of better defining the actual phenotype of patients with asthma, which may allow us to deliver targeted treatments more efficiently.

The keynote John Donaldson lecture was given by Professor M Partridge (London). He dealt with the role of the patient in asthma management and showed how patients actively want to be involved in the control of their own disease process. He focused on patient management plans and how they are now being evolved into computer-based electronic modules which patients should be able to access easily. ${ }^{40}{ }^{41}$ Critically Professor Partridge emphasised the importance of honesty and respect between both patient and physician for any management plan to work. These are lessons which can be applied to all aspects of our patient care.

\section{RESPIRATORY INFECTIONS}

In a busy symposium on mycobacterial disease, Professor P Davies (Liverpool) examined the issues surrounding treatment of tuberculosis (TB) and especially multidrug-resistant (MDR)$\mathrm{TB}$. He encouraged the use of the MDR-TB e-committee which is a UK-based group established to give advice to clinicians dealing with MDR-TB. ${ }^{42}$ This is composed of respiratory physicians, public health doctors, the reference laboratory managers and other key personnel who are happy to give their opinion and advice on new incident cases of MDR-TB via a secure blog site. In the second part of his talk, he focused on ongoing clinical trials which might in the future allow shorter duration of treatment especially with the newer fluoroquinolones such as gatifloxacin. The development of longer acting formulations of rifampicin which will allow less frequent dosage regimens and the novel antituberculous agent TMC207 are also very promising in the future treatment of this problematic worldwide disease. ${ }^{43}$

Dr A Grant (London) highlighted the fact that TB control continues to present a challenge in many resource-limited settings, particularly in southern Africa, where the overlap between $\mathrm{TB}$ and HIV infection is greatest. Innovative approaches such as projects actively seeking $\mathrm{TB}$ cases in gold mine workers in South Africa might provide lessons which may have relevance in better-resourced settings. ${ }^{44} 45$

Dr A Floto (Cambridge) gave a clear and detailed talk on recent progress in understanding the biology of infection with non-tuberculous mycobacteria (NTM). He highlighted the increasing burden of NTM and the known risk factors for infection such as underlying structural lung disease, low body mass index (BMI), gastro-oesophageal reflux and probably low vitamin $\mathrm{D}$ levels. This was reflected in a separate abstract by Ting et al, suggesting that vitamin $\mathrm{D}$ testing should be incorporated into national guidelines, at least in the paediatric population. ${ }^{46}$ Dr Floto went on to discuss how free iron may 
control growth of NTM within macrophages, how interferon $\gamma$ may restrict growth and how several metabolic processes within cells might be therapeutically manipulated to enhance intracellular killing of NTM. He ended by describing the establishment of a pan-European NTM interest group supported by the European Cystic Fibrosis Society to examine novel treatments to tackle NTM infection.

The symposium entitled 'What's new in pneumonia in adults and children' bridged the age gap and showed the similarities and differences in treating these groups for pneumonia. Professor J Simpson (Newcastle) gave an entertaining talk on the diagnosis of ventilator-associated pneumonia (VAP) and showed the potential diagnostic importance of using a battery of cytokines (including IL-1 $\beta$ and IL-8) isolated from a bronchoalveolar lavage. ${ }^{47}$ Indeed, Conway et al looked at this very group and found that in bronchoalveolar lavages in patients with VAP there were significantly higher levels of key neutrophil proteases, for example neutrophil elastase, which may also be utilised as a diagnostic marker. ${ }^{48}$ Grover et al in a similar study into VAP examined TREM-1 (triggering receptor expressed on myeloid cells-1), which is a cell surface marker of immune activation on monocytes. ${ }^{49}$ The ratio of TREM-1 concentration between lung lavage and blood was significantly higher in those patients with VAP. Both groups suggest these as potential markers of VAP and that they may play a role in its pathogenesis. In a related abstract, the topic of healthcare-associated pneumonia was explored. This is seen in patients with regular healthcare contact, and Taylor et al wished to ascertain if this was an important independent diagnostic label. ${ }^{50}$ They showed it to be common (up to $20 \%$ in their cohort of 1111 patients with respiratory infection) and on univariate analysis suggested higher mortality when compared with community-acquired pneumonia. However, when adjusted on multivariate analysis, this diagnosis did not stand as significantly associated with 30-day mortality, and as such probably offers little prognostic advantage.

Dr A Hill (Edinburgh) delivered an important talk on the use of clinical scores and focused on CURB-65 and the pneumonia severity index. ${ }^{51} \mathrm{He}$ pointed out that although these scores can help to differentiate patients that can be treated as outpatients, there are exceptions, and the scores do not work particularly well in the very young or elderly group. The importance of monitoring response to treatment was outlined by the fact that if the C-reactive protein does not fall by $>50 \%$ from baseline by day 4 after treatment there is an increased mortality at 30 days. ${ }^{52}$ He concluded that the best scoring system will probably involve the use of both clinical scores and a form of biomarker such as C-reactive protein or procalcitonin.

Moving to the paediatric patient group, Dr D Spencer (Newcastle) emphasised the importance of pneumonia in children and how this remains an important cause of worldwide childhood mortality. Although deaths from pneumonia in children in the UK are rare, there is an increasing observation of empyema and complicated pneumonia developing. ${ }^{53}$ The explanation for this is not as yet clear but could be related to the changing pneumococcal serotype prevalence ${ }^{54}$ which was expanded on by Professor D Goldblatt (London). Professor Goldblatt's talk on the pneumococcal vaccines centred on the introduction of new pneumococcal conjugate vaccines and how these have led to a reduction in the incidence of invasive pneumococcal disease. The current pneumococcal vaccine system lacks efficacy in children or elderly patients, does not stimulate immune memory and in fact on second dosing can lead to a degree of immune hyporesponsiveness. Therefore, new conjugate vaccines targeting a limited number of the capsular serotypes have been developed with improved efficacy in children. ${ }^{55}$ These conjugates are also being trialled in the elderly population to ascertain if they have a similar favourable outcome. $^{56}$

\section{THE BTS/BLF/BALR YOUNG INVESTIGATORS}

The annual Young Investigators symposium, hosted by the BTS, BLF and BALR, recognises the very best basic, translational or clinical research performed in the UK respiratory community by investigators under 35 years of age. A strong theme of this year's symposium was the exploration of novel therapeutic targets in the respiratory inflammatory response and remodelling.

Several inflammatory diseases, including COPD and asthma, have a diurnal variation in severity. Dr J Blaikley was awarded the BTS prize for his interesting work examining the role of an intrinsic cellular clock, which regulates the inflammatory response of human macrophages in a circadian manner and is dependent on a nuclear receptor Rev-erb $\alpha .{ }^{57} \mathrm{~A}$ Rev-erb $\alpha$ ligand was able to inhibit temporal inflammation and suppress genes previously implicated in the pathogenesis of COPD and asthma.

The BALR prize was awarded to Dr S Bertok for his investigation of intra-alveolar inhibition of the p55 tumour necrosis factor (TNF) receptor in ventilator-associated lung injury. ${ }^{58}$ In a murine model of ventilator-associated lung injury, significant improvements in both physiological and inflammatory parameters were demonstrated following local administration of a small domain antibody specific to the receptor.

The winner of the BLF prize, Dr MJ Heightman, provided novel data implicating tissue inhibitor of metalloproteinase-3 (TIMP-3) as an important regulator of the inflammatory response in interstitial lung disease. ${ }^{59}$ TIMP-3 is known to inhibit matrix metalloproteinases and TNF $\alpha$-converting enzyme, with suggested roles in hepatic injury and arthritis. Single nucleotide polymorphism analysis demonstrated that TIMP-3-promotor polymorphisms protected against sarcoidosis, with the protective effect associated with increased expression of TIMP-3 in alveolar and peripheral macrophages. In a bleomycin murine model, TIMP-3 knockout resulted in increased inflammation and collagen deposition.

Bafadhel et al addressed the issue of identifying the aetiology of exacerbations of COPD, which are often heterogeneous and may warrant directed treatment. ${ }^{60}$ They examined 182 COPD exacerbations, which were clinically indistinguishable, and were able to identify sputum IL-1 $\beta$, serum CXCL-10 and the percentage of blood eosinophils as biomarkers of bacteria-, virusor eosinophil-associated exacerbations, respectively. O'Reilly et al highlight that, in preschool wheezers, there is no increase in airway smooth muscle or mast cell infiltration compared with age-matched controls; thus suggesting a therapeutic window during preschool years to intervene and prevent airway remodelling. ${ }^{61}$ Chachi et al described a potential therapeutic role for restoring airway smooth muscle corticosteroid sensitivity in patients with asthma or COPD using blockade of the potassium channel KCa3.1. ${ }^{62}$

\section{RESPIRATORY RESEARCH: THE NHS, INDUSTRY AND ACADEMIA}

In recognition of the significant role the pharmaceutical industry plays in respiratory research, for the first time the BTS meeting devoted a symposium to the nature of the relationship between the NHS, academia and industry. A breadth of perspectives were represented by Professor Dame Sally Davies (Director General of Research and Development and Chief Scientific Advisor, 
Department of Health), Dr Richard Marshall (GlaxoSmithKline), Dr Gareth Rapeport (biotechnology company founder, RespiVest) and Professor Tim Higenbottam ('mid-Pharma' company, Chiesi). It was recognised that we are entering a period of change within both the pharmaceutical industry and the NHS. The pharmaceutical industry has seen a reduction in research and development spending, falling productivity and increasing cost of drug development. This is mirrored by impending cuts in NHS funding, although currently spending in health research has been maintained. Closer collaboration between the triad of NHS, industry and academia was stressed to be essential to ensure future productive respiratory research within the UK. This well received symposium addressed challenges faced in building such a collaboration, including: the need for effective, and perhaps simplified, research networks; developing research outputs that satisfy the different requirements of both industry and academia; greater information sharing and openness; training within academia and industry; and sharing of both the risk and reward.

For the Snell Memorial Lecture, the BTS were pleased to welcome a keynote speech by Professor J Drazen, the editor of the New England Journal of Medicine, on 'Lessons from clinical trials'. Professor Drazen highlighted the importance of developing a close, transparent and open relationship between physicians, regulators and the pharmaceutical industry. His message underlined the important singular doctrine that underpins our medical approach and which we should all share-Primum non nocere-or first do no harm.

\section{RESPIRATORY RESEARCH 2010 AND BEYOND}

The year 2010 was designated The Year of the Lung, by the Forum of International Respiratory Societies, to express the urgency for increased action on lung health. ${ }^{63}$ We believe this year's Winter BTS Meeting has demonstrated the ongoing excellent range and standard of clinical, translational and basic science respiratory research within the UK. Such clinical and scientific research activity will undoubtedly aid us in tackling the significant global burden of respiratory disease.

Competing interests None.

Provenance and peer review Commissioned; internally peer reviewed.

\section{REFERENCES}

1. Masters SL, Dunne A, Subramanian SL, et al. Activation of the NLRP3 inflammasome by islet amyloid polypeptide provides a mechanism for enhanced IL-1 $\beta$ in type 2 diabetes. Nat Immunol 2010;11:897-904.

2. O'Neill LA. The interleukin-1 receptor/Toll-like receptor superfamily: 10 years of progress. Immunol Rev 2008;226:10-18.

3. Reid DM, Gow NA, Brown GD. Pattern recognition: recent insights from Dectin-1. Curr Opin Immunol 2009;21:30-7.

4. Riteau N, Yazdi A, Baron L, et al. Nanoparticles cause pulmonary inflammation through IL- $1 \alpha$ and partial activation of the NLRP3 inflammasome. Thorax 2010:65(Suppl IV):A26.

5. Hammad H, Chieppa M, Perros F, et al. House dust mite allergen induces asthma via Toll-like receptor 4 triggering of airway structural cells. Nat Med 2009;15:410-16.

6. Cottey L, Jayasekera N, Haitchi H-M, et al. Airway epithelial Toll receptor expression in asthma and its relationship to disease severity. Thorax 2010;65(Suppl IV):A21.

7. Jiang D, Liang J, Fan J, et al. Regulation of lung injury and repair by Toll-like receptors and hyaluronan. Nat Med 2005:11:1173-9.

8. Jiang D, Liang J, Noble PW. Hyaluronan in tissue injury and repair. Annu Rev Cell Dev Biol 2007;23:435-61.

9. Shyamsundar M, O'Kane CM, Calfee C, et al. KGF enhances pulmonary production of pro-epithelial repair factors in a human in vivo model of acute lung injury. Thorax 2010;65(Suppl IV):A48.

10. Dancer R, Park D, D'Souza V, et al. Does vitamin D deficiency increase risk of acute lung injury post oesophagectomy? Thorax 2010;65(Suppl IV):A49.

11. Dancer R, D'Souza V, Jeffery L, et al. Is the development of acute lung injury influenced by increased levels of IL17 as a result of Treg/TH17 imbalance? Thorax 2010;65(Suppl IV):S49.
12. Calfee CS, Ware LB, Eisner MD, et al. Plasma receptor for advanced glycation end products and clinical outcomes in acute lung injury. Thorax 2008;63:1083-9.

13. Thompson B, Stitt C, McAuley D, et al. The role of the receptor for advanced end products (RAGE) in acute lung injury (ALI). Thorax 2010;65(Suppl IV):A26.

14. Zakeri N, Creagh-Brown B, Hector $L$, et al. Polymorphisms in genes encoding RAGE or RAGE ligands predispose patients to adverse outcomes following surgery necessitating cardiopulmonary bypass. Thorax 2010;65(Suppl IV):A49.

15. Palmer F, Jaffray M, Moffat $M$, et al. A historical cohort study to determine the prevalence of common chronic respiratory diseases in drug misusers. Thorax 2010;65(Suppl IV):A50.

16. Moisey EJ, Borthwick LA, Perry J, et al. Cigarette smoke dysregulates proinflammatory cytokine release from airway epithelial cells and macrophages in response to Haemophilus influenzae. Thorax 2010;65(Suppl IV):A65.

17. Comer D, Elborn J, Brown V, et al. The impact of cigarette smoke extract on inflammatory responses and Toll-like receptor-4 expression in healthy nasal epithelia cells. Thorax 2010;65(Suppl IV):A66.

18. Mackay LS, Dodd S, Tomlinson W, et al. Microvascular endothelial cell apoptosis and dysregulation of vascular endothelial growth factor receptor 2 (VEGF-R2) in response to cigarette smoke. New insights into the pathogenesis of emphysema. Thorax 2010;65(Suppl IV):A66.

19. Agusti A, Calverley PM, Celli B, et al. Characterisation of COPD heterogeneity in the ECLIPSE cohort. Respir Res 2010:11:122.

20. Han MK, Agusti A, Calverley PM, et al. Chronic obstructive pulmonary disease phenotypes: the future of COPD. Am J Respir Crit Care Med 2010;182:598-604.

21. Taylor DR. Risk assessment in asthma and COPD: a potential role for biomarkers? Thorax 2009;64:261-4.

22. Sciurba FC, Ernst A, Herth FJ, et al. A randomized study of endobronchial valves for advanced emphysema. N Engl J Med 2010;363:1233-44.

23. Tuggey JM, Plant PK, Elliott MW. Domiciliary non-invasive ventilation for recurrent acidotic exacerbations of COPD: an economic analysis. Thorax 2003;58:867-71.

24. Alam S, Li Z, Janciauskiene $S$, et al. Oxidation of $Z$ \{alpha\} 1-antitrypsin by cigarette smoke induces polymerization: a novel mechanism of early-onset emphysema. Am J Respir Cell Mol Biol Published Online First: 22 October 2010 doi:10.1165/rcmb.2010-03280C.

25. Rashid ST, Corbineau S, Hannan N, et al. Modeling inherited metabolic disorders of the liver using human induced pluripotent stem cells. J Clin Invest 2010;120:3127-36

26. Lomas DA, Parfrey H. Alpha1-antitrypsin deficiency. 4: molecular pathophysiology. Thorax 2004:59:529-35.

27. Marciniak SJ, Lomas DA. What can naturally occurring mutations tell us about the pathogenesis of COPD? Thorax 2009;64:359-64

28. Rashid M, Brown J, Ling $\mathrm{H}$, et al. Expansion of the red cell distribution width and evolving iron deficiency as predictors of poor outcome in chronic obstructive pulmonary disease. Thorax 2010;65(Suppl IV):A75.

29. McClean KM, Kee F, Young IS, et al. Obesity and the lung: 1. Epidemiology. Thorax 2008;63:649-54

30. Franssen FM, O'Donnell DE, Goossens GH, et al. Obesity and the lung: 5. Obesity and COPD. Thorax 2008;63:1110-17.

31. Collins $\mathbf{P}$, Stratton R, Kurukulaaratchy R, et al. The 'Obesity Paradox' in chronic obstructive pulmonary disease. Thorax 2010;65(Suppl IV):A74

32. Collins $\mathbf{P}$, Stratton R, Kurukulaaratchy $\mathrm{R}$, et al. Deprivation is an independent predictor of 1-year mortality in outpatients with chronic obstructive pulmonary disease. Thorax 2010:65(Suppl IV):A74.

33. Shikotra A, Hargadon B, Shelley $M$, et al. Increased expression of immunoreactive thymic stromal lymphopoetin in severe asthma. Thorax 2010;65(Suppl IV):A22.

34. Uller L, Leino $M$, Bedke $N$, et al. Double-stranded RNA induces disproportionate expression of thymic stromal lymphopoietin versus interferon-beta in bronchial epithelial cells from donors with asthma. Thorax 2010;65:626-32.

35. Farahi N, Uller L, Juss J, et al. Effects of the cyclin-dependent kinase inhibitor R-roscovitine on eosinophil survival and clearance. Thorax 2010;65(Suppl IV):A18.

36. Martin N, Arthur G, Wan W et al Suppression of constitutive and stimulated secretion of histamine from human lung mast cells by a secreted factor from lung epithelial cells. Thorax 2010;65(Suppl IV):A17.

37. Agbetile J, Fairs A, Bourne $M$, et al. Fungal sputum culture in patients with severe asthma is associated with a reduced post bronchodilator $\mathrm{FEV}_{1}$. Thorax 2010:65(Suppl IV):A61.

38. Heaney LG, Brightling CE, Menzies-Gow A, et al. Refractory asthma in the UK: cross-sectional findings from a UK multicentre registry. Thorax 2010;65:787-94.

39. Gamble J, Stevenson M, McClean E, et al. The prevalence of nonadherence in difficult asthma. Am J Respir Crit Care Med 2009;180:817-22.

40. Roberts NJ, Evans G, Blenkhorn P, et al. Development of an electronic pictorial asthma action plan and its use in primary care. Patient Educ Couns 2010;80:141-6.

41. Partridge MR, Caress AL, Brown C, et al. Can lay people deliver asthma self-management education as effectively as primary care based practice nurses? Thorax 2008:63:778-83.

42. MDR-TB Group. Clinical advisory service. 2010. http://www.brit-thoracic.org.uk/ tuberculosis/tb-links.aspx. (accessed 2011).

43. Diacon AH, Pym A, Grobusch M, et al. The diarylquinoline TMC207 for multidrug-resistant tuberculosis. N Engl J Med 2009;360:2397-405.

44. Grant AD, Coetzee L, Fielding KL, et al. 'Team up against TB': promoting involvement in Thibela TB, a trial of community-wide tuberculosis preventive therapy. AIDS 2010;24(Suppl 5):S37-44. 
45. Churchyard GJ, Fielding K, Roux S, et al. Twelve-monthly versus six-monthly radiological screening for active case-finding of tuberculosis: a randomised controlled trial. Thorax Published Online First: 23 November 2010. doi:10.1136/thx.2010.139048.

46. Ting A, Jiki M, Bell C, et al. Should routine screening for vitamin $D$ deficiency be incorporated into current guidelines for treatment of paediatric TB patients? Thorax 2010;65(Suppl IV):A15.

47. Conway Morris A, Kefala K, Wilkinson TS, et al. Diagnostic importance of pulmonary interleukin-1 beta and interleukin-8 in ventilator-associated pneumonia. Thorax 2010;65:201-7.

48. Morris AC, Kefala K, Wilkinson T, et al. Potential diagnostic significance of neutrophil proteases in ventilator-associated pneumonia. Thorax 2010;65(Suppl IV):A37.

49. Grover V, Kelleher P, Henderson D, et al. Compartmentalisation of surface triggering receptor expressed on myeloid cells-1 (TREM-1) in ventilator-associated pneumonia (VAP). Thorax 2010;65(Suppl IV):A36

50. Taylor J, Chalmers J, Singanayagam A, et al. Is healthcare associated pneumonia a distinct clinical phenotype? Thorax 2010;65(Suppl IV):A58.

51. Chalmers JD, Singanayagam A, Akram AR, et al. Severity assessment tools for predicting mortality in hospitalised patients with community-acquired pneumonia. Systematic review and meta-analysis. Thorax 2010;65:878-83.

52. Chalmers JD, Singanayagam A, Hill AT. C-reactive protein is an independent predictor of severity in community-acquired pneumonia. Am J Med 2008; 121:219-25

53. Eastham KM, Freeman R, Kearns AM, et al. Clinical features, aetiology and outcome of empyema in children in the north east of England. Thorax 2004;59:522-5.

54. Pichon B, Moyce L, Sheppard C, et al. Molecular typing of pneumococci for investigation of linked cases of invasive pneumococcal disease. J Clin Microbiol 2010;48:1926-8.
55. Koshy E, Murray J, Bottle A, et al. Impact of the seven-valent pneumococcal conjugate vaccination (PCV7) programme on childhood hospital admissions for bacterial pneumonia and empyema in England: national time-trends study, 1997-2008. Thorax 2010:65:770-4.

56. Goldblatt D, Southern J, Andrews N, et al. The immunogenicity of 7-valent pneumococcal conjugate vaccine versus 23 -valent polysaccharide vaccine in adults aged 50-80 years. Clin Infect Dis 2009;49:1318-25.

57. Blaikley J, Green L, Gibbs J, et al. Rev-erb $\alpha$, a novel anti-inflammatory target, modifies the circadian oscillation of pulmonary inflammation. Thorax 2010;65(Suppl IV):A1

58. Bertok S, Wilson M, Morley $\mathrm{P}$, et al. Blockade of intraalveolar p55 TNF-receptor signalling by a domain antibody decreases inflammation and oedema in an in vivo mouse model of ventilator-induced lung injury. Thorax 2010;65(Suppl IV):A1.

59. Heightman MJ, Booth HL, Laurent GJ, et al. Tissue inhibitor of metalloproteinase-3 (TIMP3) protects against inflammatory processes in interstitial lung disease (ILD) Thorax 2010;65(Suppl IV):A1

60. Bafadhel M, McKenna S, Terry S, et al. Acute exacerbations of chronic obstructive pulmonary disease: identification of phenotype-specific biomarkers and biological clusters. Thorax 2010;65(Suppl IV):A2.

61. O'Reilly R, Oates T, Zhu J, et al. Increased reticular basement membrane thickness but NOT airway smooth muscle in endobronchial biopsies of severe preschool wheezers. Thorax 2010;65(Suppl IV):A2.

62. Chachi L, Shikotra A, Sutcliffe A, et al. KCa3.1 ion channel blockers restore corticosteroid sensitivity in cytokine-treated airway smooth muscle (ASM) cells from both COPD and asthmatic patients. Thorax 2010;65(Suppl IV):A2

63. Forum of International Respiratory Societies. The year of the lung. 2010. http:// www.yearofthelung.org (accessed 24 Nov 2010). 